\title{
RNAi pathways in Mucor: a tale of proteins, small RNAs and functional diversity
}

\author{
Santiago Torres-Martínez and Rosa M. Ruiz-Vázquez* \\ Department of Genetics and Microbiology, Faculty of Biology, University of Murcia, \\ Murcia 30100, Spain
}

*Corresponding author:

Rosa M. Ruiz-Vázquez, Department of Genetics and Microbiology, Faculty of Biology, University of Murcia, 30100 Murcia, Spain, tel.: +34 868 887136, fax: +34 868 883963, rmruiz@um.es 


\begin{abstract}
The existence of an RNA-mediated silencing mechanism in the opportunistic fungal pathogen Mucor circinelloides was first described in the early 2000. Since then, Mucor has reached an outstanding position within the fungal kingdom as a model system to achieve a deeper understanding of regulation of endogenous functions by the RNA interference (RNAi) machinery. M. circinelloides combines diverse components of its RNAi machinery to carry out functions not only limited to the defense against invasive nucleic acids, but also to regulate expression of its own genes by producing different classes of endogenous small RNA molecules (esRNAs). The recent discovery of a novel RNase that participates in a new RNA degradation pathway adds more elements to the gene silencing-mediated regulation. This review focuses on esRNAs in $M$. circinelloides, the different pathways involved in their biogenesis, and their roles in regulating specific physiological and developmental processes in response to environmental signals, highlighting the complexity of silencing-mediated regulation in fungi.
\end{abstract}

Keywords: Mucor, RNAi, endogenous small RNAs, dicer-dependent pathway, dicerindependent pathway, mRNA degradation, gene regulation. 


\section{Introduction}

The discovery of RNA-mediated gene silencing in the late twentieth century caused a major impact in the gene expression regulation world. Conceptually described first in plants and fungi (Napoli et al., 1990; Romano and Macino, 1992), early experiments that clarified the triggering mechanism in nematodes (Fire et al., 1998) gave way to a myriad of works describing the presence of this silencing mechanism in most eukaryotic organisms (Cerutti and Casas-Mollano, 2006). The overall landscape that now draws all this information on gene silencing mechanisms is really amazing. Gene silencing was initially described as a mechanism of defense against invasive nucleic acids and viruses, with a few components participating in the machinery that generates the small interfering RNA (siRNA) molecules which are the signature of the gene silencing mechanism. In most eukaryotic systems the basic silencing machinery consists in an RNase III protein (Dicer) that processes dsRNA precursors into siRNAs, and an Argonaute protein that uses siRNAs to guide selective destruction of target mRNAs. Additionally, some silencing-competent organisms, including plants, nematodes and fungi, require an RNA-dependent RNA polymerase (RdRP) protein to generate dsRNA from single-stranded RNA inducers or to amplify siRNA signals (Ghildiyal and Zamore, 2009; Garre et al., 2014). Besides these siRNAs, multiple classes of endogenous small RNA (esRNA) molecules, including microRNAs (miRNAs) that act as riboregulators controlling a wide panoply of cellular processes, have been identified in both metazoans and lower eukaryotic organisms (Fabian et al., 2010). Biogenesis of most of those esRNAs shares a minimal common silencing machinery, named RNAi machinery, which comprises Dicer and Argonaute proteins. In addition to this basic or canonical pathway, different non-canonical alternatives in which Dicer proteins do not participate have been described. These non-canonical pathways are responsible for the biogenesis of specific esRNAs, not only the well-known Piwi-interacting RNAs (piRNAs) but also miRNAs and miRNA-like (milRNA) molecules (Senti and Brennecke, 2010; Chang et al., 2012). In these cases, the catalytic activity of Argonaute family proteins and the trimming activity of specific exonucleases are required to produce mature esRNAs. Nevertheless, most of non-canonical miRNA molecules are poorly conserved and low in abundance, which sheds doubt on their functionality. 
Among filamentous fungi, putative regulatory esRNAs produced by canonical and non-canonical pathways were first described in Neurospora crassa and Mucor circinelloides (Lee et al., 2010a; Nicolás et al., 2010). Several lines of evidences suggest that Neurospora milRNAs could regulate gene expression in a similar way to animal and plant miRNAs, although it seems that they do not play critical roles in vegetative growth or developmental processes, since Neurospora milRNA knock-out strains do not show any vegetative defect (Chang et al., 2012). However, the sterile phenotype in homozygous crosses shown by Neurospora mutants of RNAi genes required for meiotic silencing by unpaired DNA (MSUD), a RNAi-related pathway that operates during meiosis, may suggest a role of milRNAs in the regulation of sexual development (Alexander et al., 2008; Xiao et al., 2010; Hammond et al., 2011). The presence of milRNAs has been predicted in other fungi, although further functional research is necessary to evaluate their biological roles (Goodwin et al., 2011; Jiang et al., 2012; Zhou et al. 2012; Kang K et al., 2013; Lau et al. 2013; Lin et al., 2015; Dahlmann and Kück, 2015).

M. circinelloides, a basal fungus belonging to the subphylum Mucoromycotina, has achieved a major place as a fungal model system for studying different molecular processes. This has been mainly due to its distinctive position in the fungal tree of life, since this basal lineage of the fungal kingdom is evolutionary distant from other fungal models widely used for molecular studies, such as N. crassa, Aspergillus nidulans or Cryptococcus neoformans, which belong to the subkingdom Dikarya. Furthermore, Mucor is the basal fungus where more molecular tools are available, including functional analysis by RNAi. The value of $M$. circinelloides as a model has been further enhanced recently, since it has emerged as an opportunistic human pathogen, a causal agent of the rare but lethal infection mucormycosis. This emerging infection, which is caused by several fungi of the order Mucorales (Chayakulkeeree et al., 2006), mostly affects immunocompromised patients, and it presents a very high mortality rate, going up to $>90 \%$ in disseminated infections (Spellberg et al., 2005). In the last years, an increasing number of cases of mucormycosis have been described in immunocompetent/otherwise healthy individuals, which has raised the alarm on this emerging disease (Fanfair et al., 2012; Lee et al., 2014). 
M. circinelloides, which has been used as a model for studying gene silencing (Garre et al., 2014), combines basic elements of its RNAi machinery to produce different classes of esRNAs that regulate the expression of their own genes (Nicolás et al., 2010; Cervantes et al., 2013). The recent discovery of a new silencing pathway involved in the specific degradation of endogenous mRNAs, and the participation of a novel RNase III protein in this pathway (Trieu et al., 2015), adds more elements to this wide and fascinating world of gene silencing-mediated regulation. In this review we summarize our current knowledge on the pathways involved in the biogenesis of esRNA molecules and the functions which are presumably regulated by these esRNAs, providing a general overview on the regulatory role of the gene silencing machineries in significant biological processes of Mucor, including pathogenesis.

\section{Gene silencing in Mucor}

M. circinelloides is the only basal fungus in which gene silencing has been molecularly and functionally characterized, although the presence of all the key proteins of the silencing machinery in other Mucorales suggests that this mechanism may be operative in other basal fungi (Garre et al., 2014). Although the first reference on the existence of a gene silencing mechanism in Mucor dates back more than a decade (Nicolás et al., 2003), it has not been until more recently when the variety of esRNAs and the complexity of the silencing pathways involved in their biogenesis have become evident. To reach this knowledge, identification and functional characterization of the main genes involved in the gene silencing pathway induced by exogenous transgenes have been essential. Briefly, RNA-dependent RNA polymerase RdRP-1 participates in the initiation step of the silencing mechanism, since it is essential for producing antisense RNA from transgene transcripts (Calo et al., 2012). The role of RdRP-1 in gene silencing is similar to that of $N$. crassa QDE-1 protein, which is required for induction of silencing by sense transgenes (Cogoni and Macino, 1999; Goldoni et al., 2004) by generating aberrant RNA from DNA strands and converting it into dsRNA (Lee et al., 2010b). This dual polymerase activity seems to be unusual, since QDE-1 and RdRP-1 belong to the $\gamma$ family of RdRP proteins, whereas most of RdRPs from the three eukaryotic kingdoms belong to class $\alpha$ (Zong et al., 2009). Triggering dsRNA molecules produced by RdRP-1 are then processed by the main RNase III endonuclease 
Dicer-like (Dcl) protein, Dcl-2, into two different classes of siRNAs, 21 and $25 \mathrm{nt}$ long (de Haro et al., 2009). There is another Dicer enzyme, Dcl-1, which does not have a relevant role in transgene-induced silencing but is partially redundant with Dcl-2 (Nicolás et al., 2007; de Haro et al., 2009). Only one out of three Argonaute (Ago) endonucleases found in $M$. circinelloides, Ago1, is required for silencing, at least during vegetative growth, by binding siRNA molecules and provoking degradation of target transcripts (Cervantes et al., 2013). Finally, a different RdRP protein, RdRP-2, is essential for amplification of the silencing signal, a process identified in some fungi, plants and nematodes that produces new dsRNA molecules by using processed target RNAs as template (Calo et al., 2012). All these basic elements of the RNAi machinery are used by $M$. circinelloides not only to defend the genome against invasive nucleic acids but, more noteworthy, to regulate the expression of their own genes (see below) (Nicolás et al., 2010; Cervantes et al., 2013; Trieu et al., 2015).

M. circinelloides shares with many other fungi the use of this basic RNAi machinery as a defense mechanism against exogenous nucleic acid such as transgenes, viruses and transposons. Small RNA molecules derived from those inducers have been identified in several fungi and demonstrated their protective role from invasive nucleic acids, both during vegetative growth and sexual development. Characteristics of these fungal silencing pathways have been adequately reviewed elsewhere (Dang et al. 2011; Chang et al. 2012; Nicolás and Ruiz-Vázquez, 2013; Billmyre et al. 2013). Significantly, although most fungi contain several $r d r p$ genes, an amplification process producing secondary siRNAs similar to that described in M. circinelloides (Nicolás et al., 2003) has been only demonstrated in fission yeast (Simmer et al., 2010), whereas N. crassa, Aspergillus and other fungal RNAi pathways do not include amplification of the silencing signal.

\section{Endogenous dicer-dependent small RNA molecules of $M$. circinelloides}

M. circinelloides accumulates a high amount of esRNAs, which derive from exons of protein coding genes, repetitive sequences and transposons and intergenic regions (Nicolás et al., 2010). Analysis of the distribution of these esRNAs among the different types of loci revealed that they were not formed randomly across the genome, but they 
were enriched in exonic sequences compared with intergenic and repetitive regions, which is in contrast with other fungi, where most esRNAs are produced from repeats and transposons. These exon-derived esRNAs were named exonic-siRNAs (ex-siRNAs), and comparison of the ex-siRNA content in the wild type strain and RNAi mutants of $M$. circinelloides revealed that a large number of them are dicer-dependent, since they required a Dicer enzyme for their production. There are four different classes of dicerdependent ex-siRNAs, depending on the components of the silencing machinery required for their biogenesis (Nicolás et al., 2010; Cervantes et al., 2013) (Fig. 1). Class 2 is the main dicer-dependent ex-siRNAs class, and requires Dcl-2 and RdRP-1 proteins for their production. Only a small group of $d c l$-2-dependent ex-siRNAs (class 1 ) do not require RdRP-1 for their biogenesis, but most of them require RdRP-2, the protein involved in the amplification process that produces secondary siRNAs. These two exsiRNA classes specifically bind to Ago-1, one of the three $M$. circinelloides Argonaute proteins, and are accumulated at a reduced extent in ago- $1^{-}$mutants, suggesting that they require Ago-1 for their biogenesis and/or stability. Class 3 corresponds to exsiRNAs that can be generated by either Dcl-1 or Dcl-2 and require both RdRP-1 and RdRP- 2 proteins, and class 4 is a tiny group of ex-siRNAs that also require both RdRP1 and RdRP-2 and depend on $d c l-1$ but not $d c l-2$. Neither class 3 or 4 ex-siRNAs are bound by Ago-1, although they also accumulated at a reduced amount in ago- $I^{-}$mutants, suggesting that Ago-1 participates in the biogenesis of these ex-siRNA classes.

Besides the components of the silencing machinery required for their biogenesis, the different classes of dicer-dependent ex-siRNAs can also be differentiated based on specific structural features (Fig. 1). Thus, ex-siRNAs of classes 1 and 2 have a defined size of 23-24 nt with no strong strand bias, since most exons produced a mixed sense and antisense ex-siRNAs. Also, as mentioned above, they bind to Ago-1 protein, which is in agreement with a very strong bias for uracil at the 5' position of these ex-siRNAs (Nicolás et al., 2010). The 5' end of small RNAs is a crucial factor for various functional aspects of RNA silencing, including interaction with specific members of the Ago protein family (Czech and Hannon, 2011). All these features correspond to functional siRNAs produced by the canonical RNAi pathway to suppress expression of the corresponding target genes. And in fact, the regulatory role of these ex-siRNAs was experimentally validated, since the presence of specific ex-siRNAs was correlated with a decrease in mRNA accumulation of the corresponding target protein coding genes, 
whereas lack of ex-siRNAs was associated with increased accumulation of the target mRNA (Nicolás et al., 2010; Cervantes et al., 2013). All this indicates that classes 1 and 2 of ex-siRNAs are produced by a canonical dicer-dependent RNAi pathway to act as riboregulators that modulate the expression of the protein coding genes from which they are produced. In contrast, the structural characteristics of ex-siRNAs of classes 3 and 4 suggest that they are produced by a non-canonical RNA silencing mechanism. As indicated above, they do not bind to Ago-1 and show a random spread of size distribution and a very strong strand bias, since almost all of them are exclusively sense to the mRNAs (Nicolás et al., 2010; Cervantes et al., 2013). In addition, they do not present the strong bias for uracil at the 5' end shown by canonical ex-siRNAs of classes 1 and 2, although they exhibit a preference for uracil in the penultimate position for all

sizes of sRNAs. All these differential features suggest that ex-siRNAs of classes 3 and 4 are not produced by a canonical RNAi pathway, a situation often found in filamentous fungi (Lee et al., 2010a).

Endogenous sRNAs usually processed from dsRNAs by a member of the Dicer family have been also described in other fungi (Nicolás and Ruiz-Vázquez, 2013). The majority of those esRNAs derive from repeats and transposons, which may explain lack of phenotype of RNAi mutants grown under standard laboratory conditions. Besides that, a reduced number of milRNA species, produced by different biogenesis pathways, have been identified or predicted in an increasing number of ascomycetes and basidiomycetes (see section 1 for references), although lack of conservation of these milRNAs among different fungi raises doubts on their functionality. Besides $M$. circinelloides, exon-derived esRNAs with putative regulatory functions have been described in the ascomycete Trichoderma atroviride (Carreras-Villaseñor et al., 2013). Differential expression of several of those exonic-siRNAs in dicer-deficient mutants correlates with differential expression of the corresponding transcripts, suggesting that also in this fungus the RNAi pathway regulates gene expression, beyond genome protection against invasive nucleic acids.

\subsection{Target genes of dicer-dependent ex-siRNAs}

Analysis of esRNAs accumulated in $M$. circinelloides wild type strain and mutants affected in the RNAi machinery indicates that dicer-dependent ex-siRNAs are 
functional, suggesting that they could be involved in the regulation of $M$. circinelloides endogenous functions. Identification of the complete gene set regulated by the RNAi machinery is a thrilling challenge, since it can provide information on specific physiological and developmental processes controlled by this mechanism. Comparison of mRNA profiles of mutants in the RNAi pathway with that of the wild type strain is one useful approach to identify such genes. This approach not only allows to confirm the differential expression of direct targets of ex-siRNAs, but also to identify possible secondary targets, that is, genes regulated by primary or direct ex-siRNA targets. Nearly 700 genes were thus identified that are differentially expressed in silencing mutants relative to wild type (Nicolás et al., 2015). Many of those genes are up-regulated in the mutant strains, as expected for direct targets of ex-siRNAs. These include both genes from which the ex-siRNAs derive (cis regulation) and other genes with sequences completely or partially complementary to a particular ex-siRNA molecule (trans regulation). But, besides these primary targets, transcriptomic analysis also identified genes that are down-regulated in silencing mutants. They probably represent secondary targets that are controlled in trans by direct targets of ex-siRNAs. Secondary targets represent the largest proportion of genes regulated by the RNAi machinery in Mucor, suggesting that RNAi pathways control physiology and development in $M$. circinelloides by a hierarchical regulatory network in which the number of secondary targets surpasses the number of direct targets (Nicolás et al., 2015). The presence of a significant number of genes presumably involved in signal transduction or regulation among direct targets of ex-siRNAs (Nicolás et al., 2010; Cervantes et al., 2013) supports this view of amplification of gene regulation.

More than $50 \%$ of differentially regulated genes show similar expression patterns in several silencing mutants, indicating the existence of gene clusters that are similarly regulated by specific combinations of silencing proteins (Nicolás et al., 2015). Some of these gene clusters could be connected with the four different classes of dicerdependent ex-siRNAs, which require specific combinations of silencing proteins for their biogenesis (Nicolás et al., 2010; Cervantes et al., 2013) (Fig. 2). As an example, one cluster includes genes which are up-regulated in $d c l-2^{-}$, ago- $1^{-}$and $r d r p-2^{-}$mutants. This expression pattern corresponds to regulation by class 1 ex-siRNAs, which require these genes for their biogenesis. Thus, this gene cluster must include direct targets of class 1 ex-siRNAs. Similarly, other clusters were identified as grouping primary or 
secondary target genes of the different classes of dicer-dependent ex-siRNAs. The rationale is that genes misregulated in a particular combination of RNAi mutants must be controlled by ex-siRNAs that require that specific combination of silencing proteins for their biogenesis. Identification of ex-siRNA target genes allowed subsequent association of specific gene clusters with the control of specific biological responses. This was possible because, in contrast to what happens in many other fungi (Chang et al., 2012), M. circinelloides silencing mutants are affected in different biological responses.

\subsection{Physiological and developmental responses regulated by dicer-dependent ex- SiRNAs}

Phenotypes shown by $M$. circinelloides RNAi mutants are probably a consequence of altered expression of genes regulated by ex-siRNAs (Nicolás et al., 2015). Phenotypes of mutants affected in components of the silencing machinery required for biogenesis of dicer-dependent ex-siRNAs include reduced growth and altered hyphal morphology (Nicolás et al., 2007), reduced asexual sporulation (de Haro et al., 2009; Cervantes et al., 2013; Nicolás et al., 2015), and accelerated autolysis as a response to nutrient starvation (Cervantes et al., 2013). Growth and sporulation defects have been also described for RNAi mutants in some other fungi, particularly in T. atroviride, in which dicer and $r d r{ }^{-}$mutants are affected in vegetative development (Carreras-Villaseñor et al., 2013), suggesting that the silencing machinery in fungi regulates responses to environmental signals. It is worth noting that M. circinelloides ago- $1^{-}, d c l-2^{-}$and $r d r p-2^{-}$mutants, that is, those affected in components of the machinery necessary for production of class 1 ex-siRNAs, show both reduced spore production and accelerated autolysis, suggesting an interconnection between regulation of autolysis and sporulation. Vegetative growth, hyphal morphology, sporulation and autolysis are developmental responses to endogenous and environmental signals in which many genes are involved and the existence of common regulatory networks cannot be discarded. In fact, it has been demonstrated that genes that control asexual sporulation in A. nidulans also regulate hyphal autolysis (Emri et al., 2008; Kang JY et al., 2013). The evidence that the RNAi machinery could have a prominent role in the regulatory pathway that controls these two processes in $M$. circinelloides is an exciting possibility. The diversity in mutant phenotypes found in $M$. circinelloides silencing mutants highlights the importance of 
the RNAi machinery in endogenous regulation of complex developmental processes, modulating the expression of genes involved in these responses. A remarkable example of the $M$. circinelloides ability to adapt to the changing environment by using the RNAi machinery to manage a response has been recently reported (Calo et al., 2014). Mucor is able to develop spontaneous resistance to the antifungal drug FK506 via two distinct mechanisms. Some of the spontaneous resistant isolates present mutations in genes coding for FK506 targets that confer stable drug resistance. But, in addition, a number of unstable FK506 resistant strains can be isolated which harbored no mutations in any of those genes. Authors demonstrated that this resistance arises through a novel epigenetic mechanism in which RNAi is spontaneously triggered to silence the drug target gene $f k b A$ giving rise to drug-resistant epimutants (Calo et al., 2014). This resistance is unstable, since those epimutants reverted to the drug-sensitive wild type phenotype when grown in the absence of drug. The possibility that such epigenetic mechanism promoting phenotypic adaptations may also work in other eukaryotes is certainly suggestive.

Each one of the physiological and developmental responses regulated by the RNAi machinery is altered in specific combinations of silencing mutants. Thus, by contrasting genes misregulated in a particular group of mutants with the specific phenotypes shown by those mutants, a connection could be established among specific gene clusters and specific biological responses. The asexual spore production in $M$. circinelloides is a good example. This process is controlled mainly by $d c l-2$, ago-1 and $r d r p-2$ genes and, as mentioned above, the same set of genes is also involved in accelerated autolysis (de Haro et al., 2009; Cervantes et al., 2013; Nicolás et al., 2015), suggesting that both processes could be controlled by genes regulated directly or indirectly by class 1 ex-siRNAs. In fungi, both cellular processes are connected with nutrient sensing of the cells, particularly carbon source levels in the medium (Steyaert et al., 2010; Park and Yu, 2012). Therefore, class 1 ex-siRNAs may participate in modulating expression of genes involved in nutrient sensing pathways. The mRNA transcriptome analysis identified a number of genes with expression patterns corresponding to regulation by class 1 ex-siRNAs. This group of genes includes much more genes than those initially identified as producers of class 1 ex-siRNAs (Nicolás et al., 2010; Nicolás et al., 2015), suggesting an amplified regulatory mechanism that operates in asexual spore production and/or autolysis, with genes acting at different 
regulatory levels. The putative functions of several of these genes regulated by $d c l-2$, ago- 1 and $r d r p-2$, presumably through the action of class 1 ex-siRNAs, may explain the defects in asexual spore production or/and accelerated autolysis in response to nutrient signals shown by the corresponding null mutants (Nicolás et al., 2015). Thus, several of those genes code for proteins involved in signal transduction or regulation and in specific metabolic pathways that have been associated with asexual sporulation and/or autolysis in other fungi, such as proteins involved in $\mathrm{G}$ protein signaling (Pérez and Cansado, 2010; Park and Yu, 2012), methyltransferases (Sarikaya-Bayram et al., 2015), or lipid metabolism (Carreras-Villaseñor et al., 2013; Ivell et al., 2013). Subsequent detailed and individual functional analyses of identified genes would allow recognition of the particular ones responsible for this phenotype related with nutrient sensing.

One particularly interesting phenotype shown by specific RNAi mutants is the defect in zygospore production during the sexual cycle shown by $r d r p-I^{-}$and $r d r p-2^{-}$ mutants (Nicolás et al., 2015). Zygospores are sexual structures resulting from the fusion of specialized hyphae (zygophores) of two different mating types. Sexual interaction is mediated by a mucoralean specific pheromone, trisporic acids, which trigger the formation of zygophores in the contact zone (Schimek and Wöstemeyer, 2009). Compared with the wild type and the remaining silencing mutants, $r d r p-l^{-}$and $r d r p-2^{-}$mutants produce less zygospores although with a wild type morphology, suggesting that the RNAi machinery could regulate initial steps of sexual interaction. Interestingly, transcriptome data identify clusters of genes which are specifically misregulated in $r d r p-1^{-}$and/or $r d r p-2^{-}$mutants but not in other RNAi mutants (Fig. 2). One of those genes encodes a protein highly similar to the oligopeptide transporter protein ISP4 of Schizosaccharomyces pombe, which is involved in the sexual differentiation process (Sato et al., 1994). All these results suggest that $r d r p-1$ and $r d r p$ 2 should participate in the biogenesis of a different class of small RNA that would be produced by a dicer-independent mechanism. These small RNAs would specifically regulate genes involved in sexual interaction and production of zygospores, as well as genes involved in the responses to other environmental signals that are specifically affected in these two mutants, such as the response to oxidative stress or the ability to grow at low pH (Nicolás et al., 2015; Trieu et al., 2015). 


\section{The dicer-independent pathway}

Besides the canonical dicer-dependent esRNAs, deep sequencing of small RNAs (18$25 \mathrm{nt}$ ) accumulated by $M$. circinelloides wild type and RNAi mutant strains identified more than 500 new loci that produced esRNAs by a dicer-independent but $r d r p-1$ and/or $r d r p$-2-dependent mechanism. That is, they are accumulated at a reduced amount in either or both $r d r p^{-}$mutants relative to wild type and dicer mutant strains (Trieu et al., 2015). Most of these $r d r p$-dependent dicer-independent esRNAs correspond to exons and, surprisingly, they present structural characteristics that are shared by the dicerdependent class 3 ex-siRNAs, suggesting the existence of common elements involved in the biogenesis of these dicer-independent and dicer-dependent esRNAs (Nicolás et al., 2010; Trieu et al., 2015). In fact, rdrp-dependent dicer-independent esRNAs have a very strong strand bias, almost all of them being exclusively sense to the mRNAs, and show a random spread of size distribution without enrichment for a specific size, suggesting that they are small mRNA degradation products. It has been proposed that these $r d r p$-dependent dicer-independent esRNAs are produced by a non-canonical degradation pathway, where the RdRP proteins signal specific transcripts for degradation by a newly identified RNase III-like protein, named R3B2. This ribonuclease should preferentially cleave mRNA two nucleotides downstream of any uracil, since $r d r p$-dependent dicer-independent esRNAs show a strong bias for uracil at the penultimate position for all sizes of these esRNAs (Trieu et al., 2015). According to the nature of these esRNAs, which corresponds to non-random degradation products of mRNAs that are generated by an $r d r p$-dependent mechanism, they were named as $r d r p$ dependent degraded RNA (rdRNA).

The fact that levels of rdRNAs were significantly reduced in $r d r p^{-}$mutants relative to wild type and dicer strains supported the proposal that they were not random degradation products, thus raising the possibility that this new non-canonical pathway could also regulate mRNA levels. And in fact, the $r d r p$-dependent dicer-independent RNA degradation pathway regulates gene expression, since reduction in rdRNA levels in $r d r p^{-}$mutants correlates with an increase in mRNA accumulation of the corresponding genes (Trieu et al., 2015). This confirmed the existence of a degradation pathway in $M$. circinelloides that regulates mRNA levels and requires RdRP-1 and/or RdRP-2 proteins but not Dcl-1 and Dcl-2. Among genes regulated by this non-canonical 
pathway the most highly enriched class was the coenzyme transport and metabolism category, which includes 12 genes that participate in heme B biosynthesis or metabolism (Franken et al, 2011; Trieu at al., 2015). Five out of eight proteins involved in heme B biosynthetic pathway, as well as proteins involved in heme metabolism, are regulated by the $r d r p$-dependent dicer-independent degradation pathway, suggesting a role for this pathway in the regulation of heme-containing protein(s). Impairment of the degradation pathway in $r d r p^{-}$mutants would result in an increased accumulation of mRNAs corresponding to the mentioned genes and, consequently, an increase of intracellular heme levels. In fungi, hemes are found in a number of biological relevant proteins, such as peroxidases, cytochrome, flavohemoglobins and others (Franken et al., 2011). Many of these proteins are involved in the response to different environmental stresses, such as low oxygen conditions (Hillmann et al., 2014). Interestingly, one of the most relevant fungal hemoproteins is catalase, which is essential for protecting the cell from oxidative damage (Hansberg et al., 2012). Several other genes involved in the antioxidant response are also regulated by the $r d r p$-dependent dicer-independent degradation pathway (Trieu et al., 2015). Up regulation of those genes in $r d r p^{-}$mutants relative to wild type, together with the increase in heme biosynthesis, could be responsible for the specific phenotypic alterations shown by those mutants, which are more resistant to oxidative stress than the wild type strain (Trieu et al., 2015). On the other hand, the complexity of the phenotype related to altered sexual behavior and reduced production of zygospores shown by $r d r p^{-}$mutants, and the high number of genes regulated by the rdrp-dependent dicer-independent pathway containing domains involved in transcriptional regulation or signal transduction, have made it difficult to associate specific genes with this phenotype. However, one of the genes regulated by this pathway codes for a protein highly similar to the mating factor M secretion protein Mam1 of $S$. pombe, which is responsible for secretion of the mating pheromone (Christensen et al., 1997). It has been suggested that modulation of expression of the $M$. circinelloides protein in $r d r p^{-}$mutants could be responsible, at least in part, for the defects shown by those mutants in their sexual behavior (Trieu et al., 2015).

\subsection{R3B2, a new RNase III protein}

The pathway for the production of rdRNAs required the participation of an RNase to degrade target mRNAs. By searching the $M$. circinelloides genome (v2.0) for candidate 
RNases to be involved in this non-canonical pathway and after functional analysis of the corresponding knockout mutants, a protein containing an RNase III-like domain (r3) and two dsRNA binding domain (b2) was identified and named R3B2 (Trieu et al., 2015). Evidences for the involvement of $r 3 b 2$ gene in the non-canonical RNA degradation pathway were provided by mRNAs accumulation analysis of representative rdRNA-producing exons. In all cases, mRNAs produced from those loci were upregulated both in $r 3 b 2^{-}$and $r d r p-1^{-}$and/or $r d r p-2^{-}$mutants compared to wild-type strain and dicer mutant (Trieu et al., 2015), which is the expected result if $r 3 b 2$ gene encodes the RNase required for degradation of specific mRNAs by the $r d r p$-dependent, dicerindependent non-canonical pathway. A further confirmation on the role of RNase III R3B2 in the generation of rdRNAs by the $r d r p$-dependent dicer-independent pathway came from deep sequencing analysis of the esRNA content in $r 3 b 2^{-}$mutant and its comparison with wild type strain. More than 1500 exonic loci showing a significant reduction in normalized esRNA reads in $r 3 b 2^{-}$mutant relative to wild type were identified. These loci included all but one of rdRNA-producing loci, pointing to a relevant role of R3B2 in the $r d r p$-dependent dicer-independent degradation pathway (Trieu et al., 2015). Surprisingly, also a significant number of dicer-dependent exsiRNA loci, mainly those belonging to class 3 ex-siRNAs, were found to be dependent on R3B2 for their biogenesis (see below).

The domain architecture of the R3B2 protein is rather unusual, since prokaryotic and fungal class 1 RNase IIIs contain only one dsRNA binding domain (dsRBD), in addition to the RNase III catalytic motif, whereas classes 2 and 3 of eukaryotic RNase IIIs are larger proteins with several structural domains, as occurs in Drosha and Dicer (MacRae and Doudna, 2007). Similar to class 1 RNase IIIs, R3B2 contains a single RNase III-like domain, which shows several differences in conserved residues relative to the RNase III family signature. Despite these differences, the RNase III-like domain of R3B2 is required for its efficient function in the non-canonical RNA degradation pathway, since mutant alleles affected in specific residues of this domain fail to complement lack of R3B2 function in the $r 3 b 2^{-}$mutant (Trieu et al., 2015). In addition to the single RNase III-like domain, R3B2 contains two dsRNA binding domains. As indicated before, this overall domain organization is not found in any of the common RNase III families. Only a single protein from Arabidopsis thaliana, the RNase III-like protein 2 (AtRTL2), displays this unusual domain organization (Comella et al., 2008), 
although sequence similarity between this protein and $M$. circinelloides R3B2 is low. The AtRTL2 protein cleaves dsRNAs in vitro, and is involved in the production of small RNAs derived from transgenes in vivo, probably by interacting with other $A$. thaliana Dicer enzymes to positively affect Dicer activity in siRNA generation (Kiyota et al., 2011). The similar and unique domain organization shared by AtRTL2 and R3B2 could suggest that the two dsRBD might be utilized by Mucor R3B2 for protein-protein interactions with other members of the RNAi machinery (e.g. RdRP or Dicer proteins), to degrade target transcripts or positively affect siRNA production (Trieu et al., 2015). Strikingly, proteins similar to R3B2 have been specifically found only in the mucoralean basal lineage of the fungal kingdom, suggesting that R3B2 belongs to an RNase family specific to Mucorales (Trieu et al., 2015). The RNase III-like domain of R3B2 shows a limited similarity with RNase III domains of bacteria of the order Burkholderiales ( $\beta$-proteobacteria), which suggests that this protein family could derive from a horizontal transfer event between Burkholderia and an ancestor of the order Mucorales (Trieu et al., 2015).

\subsection{R3B2 and the canonical dicer-dependent RNA silencing pathway}

The role of $\mathrm{R} 3 \mathrm{~B} 2$ is not limited to its participation in the rdrp-dependent dicerindependent degradation pathway. A significant number of dicer-dependent ex-siRNAs, mainly those belonging to class 3, are also found among esRNAs significantly downregulated in $r 3 b 2^{-}$mutant relative to wild type, indicating that this ex-siRNA class and, to a lesser extent, other dicer-dependent ex-siRNA classes, require R3B2 for their biogenesis (Trieu et al., 2015). Participation of R3B2 in class 3 ex-siRNA biogenesis is not surprising, since, as mentioned above, class 3 ex-siRNAs share several structural and functional features with $r d r p$-dependent dicer-independent rdRNAs, including their inability to be bound by Ago1 (Nicolás et al., 2010; Cervantes et al., 2013; Trieu et al., 2015). It had been proposed that class 3 ex-siRNAs could be produced by degradation of specific mRNAs by unknown RNases (Nicolás et al., 2010; Cervantes et al., 2013). Their similarities to rdRNAs suggest that these ex-siRNAs are also degradation products generated by R3B2 through a non-canonical RNAi pathway. This pathway would be different to the rdrp-dependent dicer-independent one, since either Dcl-1 or Dcl-2 is required for biogenesis of class 3 ex-siRNAs (Trieu et al., 2015). Thus, R3B2 could participate in several RNA degradation pathways that regulate different groups of 
genes and in which participate different combinations of silencing proteins. These pathways could share some steps, such as the activity of RdRP-1 and/or RdRP-2 on target transcripts, which would generate discrete dsRNA stretches that could be directly recognized by the RNase III-like R3B2, targeting those transcripts for degradation (rdRNAs), or could be firstly processed by either Dcl-1 or Dcl-2 and after the initial cleavage the single stranded portions of mRNAs would be degraded by R3B2 (class 3 ex-siRNAs) (Trieu et al., 2015).

Besides the function of R3B2 in generation of rdRNAs and class 3 ex-siRNAs, this protein also participates in the biogenesis of a significant number of dicerdependent ex-siRNAs of class 2 (Trieu et al., 2015). The particular function of R3B2 in the biogenesis of these canonical ex-siRNAs is not known, although the presence of a functional RNase III-like domain is absolutely required, since mutants expressing a $\mathrm{R} 3 \mathrm{~B} 2$ protein with amino acid substitution in conserved positions of the RNase III domain fail to activate the canonical dicer-dependent RNAi pathway (Trieu et al., 2015). It is possible that, similarly to A. thaliana AtRTL2, R3B2 protein may interact with Dcl2 to positively affect the Dicer activity in siRNA generation (Kiyota et al., 2011).

\subsection{Biological processes regulated by $\mathrm{R} 3 \mathrm{~B} 2$}

As expected from the participation of $\mathrm{R} 3 \mathrm{~B} 2$ in the canonical dicer-dependent RNAi pathway and non-canonical $r d r p$-dependent RNA degradation pathway, the $r 3 b 2^{-}$ mutant presents phenotypes associated with alterations in cellular processes controlled by those pathways (Trieu et al., 2015). Thus, processes connected with nutrient sensing, such as production of vegetative spores and autolysis induced by nutrient starvation, which are regulated by the dicer-dependent canonical pathway, are affected in the null $r 3 b 2^{-}$mutant. In addition to that, this mutant also shows an increased resistance to oxidative stress, similar to that of $r d r p^{-}$mutants, and a reduction in sexual interaction and production of zygospores. These two physiological and developmental processes are regulated by the $r d r p$-dependent dicer-independent RNA degradation pathway, which highlights the relevant role of R3B2 in this non-canonical pathway. Genes controlled by either dicer-dependent or $r d r p$-dependent dicer-independent pathways are included within r3b2-dependent esRNA producing loci (Trieu et al., 2015). Modulation of gene expression in the $r 3 b 2^{-}$mutant of some of those genes could be then responsible 
of the defects shown by this mutant in biological processes participated by the $r 3 b 2$ gene.

A large number of loci producing r $3 b 2$-dependent esRNAs remain without assignment to any known RNAi pathway, suggesting that R3B2 actively regulates the expression of a broad set of genes that could be involved in different processes, including pathogenesis. The possibility that R3B2 and other components of the RNAi machinery could have a role in Mucor pathogenesis has been explored by using a mouse model to investigate host-pathogen interactions (our unpublished results). Immunosuppressed mice, as well as the invertebrate Galleria mellonella, have been previously used as hosts to identify virulence factors in Mucor pathogenesis. These studies show that virulence differences among distinct Mucor species are linked to spore size, the large spores associated to (-) mating type showing higher virulence than small spores produced by (+) mating type (Li et al., 2011). Although the molecular bases of this size dimorphism are unknown, the calcineurin signaling pathway seems to be involved (Lee et al., 2013). This pathway also regulates yeast-mold dimorphic transition in Mucor, a process which is also related to virulence (Lee et al., 2013; Lee et al., 2015). Besides these virulence factors, our preliminary results suggest that the $r d r p$ dependent dicer-independent degradation pathway may regulate genes with a role in pathogenesis, since mutants affected in this pathway show a differential virulence relative to wild type (our unpublished results). To better understand how gene expression is regulated during infection, a zebrafish (Danio rerio) model for Mucor pathogenesis analysis has been developed and used to investigate how esRNA and mRNAs profiles of both pathogen and host change during the infection process. A significant number of genes differentially expressed during infection of zebrafish by virulent and avirulent $M$. circinelloides strains have been identified (our unpublished results). Detailed analysis of those genes will be necessary to reveal their role in Mucor pathogenesis and the real regulatory dimension of this new RNA degradation-based mechanism.

\section{A genetic link between mRNA degradation and post-transcriptional RNA silencing}


The different silencing pathways identified in $M$. circinelloides regulate different groups of genes (Nicolás et al., 2010; Trieu et al., 2015). This can be deduced not only from the specific phenotypes shown by different silencing mutants but also from transcriptomic analysis data (Nicolás et al., 2015), which identify clusters of genes differentially regulated in specific combination of silencing mutants. In summary, two major silencing pathways have been identified in $M$. circinelloides to regulate gene expression in different ways (Fig. 3). The dicer-dependent RNAi pathway produces ex-siRNAs that regulate gene expression by binding to Ago proteins and provoking degradation of target mRNAs. The main components of the canonical RNAi machinery, that is, Dicer, Ago and RdRP proteins, participate in this pathway. The other major pathway is the rdrp-dependent dicer-independent one, which is really a degradation pathway in which participate specific components of the RNAi machinery, the RdRP proteins. This pathway regulates gene expression by degradation of specific transcripts by the newly identified RNase III protein R3B2, which seems to be specific of mucoralean fungi. It has been speculated that the $r d r p$-dependent dicer-independent degradation pathway may represent the first evolutionary step in the development of the canonical RNAi mechanism (Trieu et al., 2015). RNAi is a complex process and it is unlikely that the entire process developed at once. The RdRP proteins, which represent a genetic link between mRNA degradation and post-transcriptional silencing, could be the first players that somehow marked mRNAs for degradation. This ancestral RNA degrading mechanism could have evolved to generate RNAi and its different pathways and functional diversifications. Thus, Dicer may have appeared and cleaved the RdRP products and later on Argonaute proteins would have evolved to acquire siRNAs produced by Dicer and use them for further RNA degradation. It is tempting to speculate that in $M$. circinelloides, and probably other members of the mucoralean basal lineage of the fungal kingdom, both, the ancient and the evolved mechanisms are still simultaneously operating. 


\section{Acknowledgments}

Work on RNAi pathways in Mucor in our laboratory is supported by the Spanish

Ministerio de Educación y Ciencia (BFU2006-02408 and GEN2006-28558-E),

Ministerio de Ciencia e Innovación (BFU2009-07220) and Ministerio de Economía y Competitividad (BFU2012-32246), Fundación Séneca de la Comunidad Antónoma de la Región de Murcia, Spain (19339/PI/14) and the DOE Joint Genome Institute, USA (DE-ACO2-05CH11231). 


\section{Figure Legends}

Fig. 1. Proteins involved in the biogenesis of the four classes of dicer-dependent exsiRNAs in M. circinelloides. Number of exonic loci belonging to each class is indicated inside boxes. Blue circles show the involvement of different RNAi enzymes in the biogenesis of each ex-siRNA class. Half circles in class 3 biogenesis indicate the redundant role of Dcl-1 and Dcl-2. The main structural features of each ex-siRNA class are indicated. Sense and antisense strands of ex-siRNAs are depicted in blue and red colors, respectively.

Fig. 2. Heat map depicting gene expression in RNAi mutants. Genes with similar expression patterns in the indicated mutants have been clustered and correlated with different ex-siRNA classes, which require particular combination of RNAi proteins for their biogenesis. Red and green colors represent down- and up-regulation, respectively. Only some of the clusters identified have been represented. The dicer-dependent gene clusters 1, 2 and 3 and dicer-independent cluster 4 are shown. Figure modified from Nicolás et al. (2015).

Fig. 3. Two main RNA silencing pathways in M. circinelloides. The canonical dicerdependent RNAi pathway (left) produces ex-siRNAs belonging to classes 1 and 2 . These ex-siRNA classes are generated by Dcl-2 from dsRNA molecules derived from cellular transcripts by the action of RdRP-1 (class 2) or RdRP-2 (class 1). The RNase III-like protein R3B2 plays a role in the production of some of these ex-siRNAs, but its specific function is not yet known. Classes 1 and 2 are canonical ex-siRNAs with a defined size of 23-24 nt and a preference for uracil at the 5' position. These ex-siRNAs are bound to Ago-1 and used to identify target mRNAs and mediate their degradation. The $r d r p$-dependent dicer-independent pathway (right) produces rdRNAs derived from specific transcripts that are targeted for degradation by RdRP-1 and/or RdRP-2 binding. The RdRP proteins may be able to make a short complementary strand that signals R3B2 for degradation. This protein preferentially cleaves mRNAs two nucleotides downstream of any uracil, giving rise to different size fragments with a uracil in the penultimate position (rdRNAs). Biogenesis of dicer-dependent class 3 ex-siRNAs (not represented) may be similar to the rdRNAs, but the discrete dsRNA stretches synthetized by RdRP proteins would be first processed by either Dcl-1 or Dcl-2 and 
then the single-stranded portions of mRNAs generated by this initial cleavage would be degraded by R3B2. Structural characteristics of class 3 ex-siRNAs are very similar to that of rdRNAs. Figure modified from Trieu et al. (2015). 


\section{References}

Alexander, W.G., Raju, N.B., Xiao, H., Hammond, T.M., Perdue, T.D., Metzenberg, R.L., Pukkila, P.J., Shiu, P.K., 2008. DCL-1 colocalizes with other components of the MSUD machinery and is required for silencing. Fungal Genet. Biol. 45, 719-727.

Billmyre, R.B., Calo, S., Feretzaki, M., Wang, X., Heitman, J., 2013. RNAi function, diversity, and loss in the fungal kingdom. Chromosome Res. 21, 561-572.

Calo, S., Nicolas, F.E., Vila, A., Torres-Martinez, S., Ruiz-Vazquez, R.M., 2012. Two distinct RNA-dependent RNA polymerases are required for initiation and amplification of RNA silencing in the basal fungus Mucor circinelloides. Mol. Microbiol. 83, 379394.

Calo, S., Shertz-Wall, C., Lee, S.C., Bastidas, R.J., Nicolás, F.E., Granek, J.A., Mieczkowski, P., Torres-Martínez, S., Ruiz-Vázquez, R.M., Cardenas, M.E., et al., 2014. Antifungal drug resistance evoked via RNAi-dependent epimutations. Nature 513, $555-558$.

Carreras-Villaseñor, N., Esquivel-Naranjo, E.U., Villalobos-Escobedo, J.M., AbreuGoodger, C., Herrera-Estrella, A., 2013. The RNAi machinery regulates growth and development in the filamentous fungus Trichoderma atroviride. Mol. Microbiol. 89, 96112.

Cerutti, H., Casas-Mollano, J.A., 2006. On the origin and functions of RNA-mediated silencing: from protists to man. Curr. Genet. 50, 81-99.

Cervantes, M., Vila, A., Nicolás, F.E., Moxon, S., de Haro, J. P., Dalmay, T., TorresMartínez, S., Ruiz-Vázquez, R.M., 2013. A single argonaute gene participates in exogenous and endogenous RNAi and controls cellular functions in the basal fungus Mucor circinelloides. PLoS One, 8, e69283. doi: 10.1371/journal.pone.0069283.

Chang, S.S., Zhang, Z., Liu, Y., 2012. RNA interference pathways in fungi: mechanisms and functions. Annual Rev. Microbiol. 66, 305-323. 
Chayakulkeeree, M., Ghannoum, M.A, Perfect, J.R., 2006. Zygomycosis: the reemerging fungal infection. Eur. J. Clin. Microbiol. Infect. Dis. 25, 215-229.

Christensen, P.U., Davey, J., Nielsen, O., 1997. The Schizosaccharomyces pombe mam1 gene encodes an $\mathrm{ABC}$ transporter mediating secretion of M-factor. Mol. Gen. Genet. $255,226-236$.

Cogoni, C., Macino, G., 1999. Gene silencing in Neurospora crassa requires a protein homologous to RNA-dependent RNA polymerase. Nature 399, 166-169.

Comella, P., Pontvianne, F., Lahmy, S., Vignols, F., Barbezier, N., Debures, A., Jobet, E., Brugidou, E., Echeverria, M., Sáez-Vásquez, J., 2008. Characterization of a ribonuclease III-like protein required for cleavage of the pre-rRNA in the 3'ETS in Arabidopsis. Nucleic Acids Res. 36, 1163-1175.

Czech, B., Hannon, G.J., 2011. Small RNA sorting: matchmaking for Argonautes. Nat. Rev. Genet. 12, 19-31.

Dahlmann, T.A., Kück, U., 2015. Dicer-dependent biogenesis of small RNAs and evidence for microRNA-like RNAs in the penicillin producing fungus Penicillium chrysogenum. PLoS One 10, e0125989. doi: 10.1371/journal.pone.0125989.

Dang, Y., Yang, Q., Xue, Z., Liu, Y., 2011. RNA Interference in Fungi: Pathways, Functions, and Applications. Eukaryot. Cell 10, 1148-1155.

de Haro, J.P., Calo, S., Cervantes, M., Nicolás, F.E., Torres-Martínez, S., Ruiz-Vázquez, R.M., 2009. A single dicer gene is required for efficient gene silencing associated with two classes of small antisense RNAs in Mucor circinelloides. Eukaryot. Cell 8, 14861497.

Emri, T., Molnár, Z., Szilágyi, M., Pócsi, I., 2008. Regulation of autolysis in Aspergillus nidulans. Appl. Biochem. Biotech. 151, 211-220. 
Fabian, M.R., Sonenberg, N., Filipowicz, W., 2010. Regulation of mRNA translation and stability by microRNAs. Annual Rev. Biochem. 79, 351-379.

Fanfair, R.N., Benedict, K., Bos, J., Bennett, S.D., Lo, Y.C., Adebanjo, T., Etienne, K., Deak, E., Derado, G., Shieh, W.J., et al., 2012. Necrotizing cutaneous mucormycosis after a tornado in Joplin, Missouri, in 2011. N. Engl. J. Med. 367, 2214-2225.

Fire, A., Xu, S., Montgomery, M.K., Kostas, S.A., Driver, S.E., Mello, C.C., 1998. Potent and specific genetic interference by double-stranded RNA in Caenorhabditis elegans. Nature 391, 806-811.

Franken, A.C., Lokman, B.C., Ram, A.F., Punt, P.J., van den Hondel, C.A., de Weert, S., 2011. Heme biosynthesis and its regulation: towards understanding and improvement of heme biosynthesis in filamentous fungi. Appl. Microbiol. Biotechnol. 91, 447-460.

Garre, V., Nicolás, F.E., Torres-Martínez, S., Ruiz-Vázquez, R.M., 2014. The RNAi Machinery in Mucorales: The Emerging Role of Endogenous Small RNAs, in: Sesma, A., von der Haar, T. (Eds.), Fungal RNA Biology. Springer International Publishing, Switzerland, pp. 291-313.

Ghildiyal, M., Zamore, P.D., 2009. Small silencing RNAs: An expanding universe. Nat. Rev. Genet. 10, 94-108.

Goldoni, M., Azzalin, G., Macino, G., Cogoni, C., 2004. Efficient gene silencing by expression of double stranded RNA in Neurospora crassa. Fungal Genet. Biol. 41, 1016-1024.

Goodwin, S.B., Ben M'Barek, S., Dhillon, B., Wittenberg, A.H.J., Crane, C.F., Hane, J.K., Foster, A.J., Van der Lee, T.A.J., Grimwood, J., Aerts, A., et al., 2011. Finished genome of the fungal wheat pathogen Mycosphaerella graminicola reveals dispensome structure, chromosome plasticity, and stealth pathogenesis. PLoS Genet. 7, e1002070. doi: 10.1371/journal.pgen.1002070. 
Hammond, T.M., Xiao, H., Boone, E.C., Perdue, T.D., Pukkila, P.J., Shiu, P.K.T, 2011. SAD-3, a putative helicase required for meiotic silencing by unpaired DNA, interacts with other components of the silencing machinery. G3: Genes, Genomes, Genetic 1, 369-376.

Hansberg, W., Salas-Lizana, R., Domínguez, L., 2012. Fungal catalases: function, phylogenetic origin and structure. Arch. Biochem. Biophys. 525, 170-180.

Hillmann, F., Linde, J., Beckmann, N., Cyrulies, M., Strassburger, M., Heinekamp, T., Haas, H., Guthke, R., Kniemeyer, O., Brakhage, A.A., 2014. The novel globin protein fungoglobin is involved in low oxygen adaptation of Aspergillus fumigatus. Mol. Microbiol. 93, 539-553.

Ivell, R., Balvers, M., Anand, R.J.K., Paust, H., McKinnell, C., Sharpe, R., 2013. Differentiation-dependent expression of $17 \beta$-hydroxysteroid dehydrogenase, type 10, in the rodent testis: effect of aging in Leydig cells. Endocrinology 144, 3130-3137.

Jiang, N., Yang, Y., Janbon, G., Pan, J., Zhu, X., 2012. Identification and functional demonstration of miRNAs in the fungus Cryptococcus neoformans. PLoS One 7, e52734. doi: 10.1371/journal.pone.0052734.

Kang, K., Zhong, J., Jiang, L., Liu, G., Gou, C.Y., Wu, Q., Wang, Y., Luo, J., Gou, D., 2013. Identification of microRNA-like RNAs in the filamentous fungus Trichoderma reesei by Solexa Sequencing. PLoS One 8, e76288. doi: 10.1371/journal.pone.0076288.

Kang, J.Y., Chun, J., Jun, S.C., Han, D.M., Chae, K.Y., 2013. The MpkB MAP kinase plays a role in autolysis and conidiation of Aspergillus nidulans. Fungal Genet. Biol. 61, $42-49$.

Kiyota, E., Okada, R., Kondo, N., Hiraguri, A., Moriyama, H., Fukuhara, T., 2011. An Arabidopsis RNase III-like protein, AtRTL2, cleaves double-stranded RNA in vitro. J. Plant Res. 124, 405-414. 
Lau, S.K., Chow, W.N., Wong, A.Y., Yeung, J.M., Bao, J., Zhang, N., Lok, S., Woo, P.C., Yuen, K.Y., 2013. Identification of microRNA-like RNAs in mycelial and yeast phases of the thermal dimorphic fungus Penicillium marneffei. PLoS Negl. Trop. Dis. 7, e2398. doi: 10.1371/journal.pntd.0002398.

Lee, H.C., Li, L., Gu, W., Xue, Z., Crosthwaite, S.K., Pertsemlidis, A., Lewis, Z.A., Freitag, M., Selker, E.U., Mello, C.C., et al., 2010a. Diverse pathways generate microRNA-like RNAs and Dicer-independent small interfering RNAs in fungi. Mol. Cell 38, 803-814.

Lee, H.C., Aalto, A.P., Yang, Q., Chang, S.S., Huang, G., Fisher, D., Cha, J., Poranen, M.M., Bamford, D.H., Liu, Y., 2010b. The DNA/RNA-dependent RNA polymerase QDE-1 generates aberrant RNA and dsRNA for RNAi in a process requiring replication protein A and a DNA helicase. PLoS Biol. 8, e1000496. doi: 10.1371/journal.pbio.1000496.

Lee, S.C., Li, A., Calo, S., Heitman, J., 2013. Calcineurin plays key roles in the dimorphic transition and virulence of the human pathogenic zygomycete Mucor circinelloides. PLoS Pathog. 9, e1003625. doi: 10.1371/journal.ppat.1003625.

Lee, S.C., Billmyre, R.B., Li, A., Carson, S., Sykes, S.M., Huh, E.Y., Mieczkowski, P., Ko, D.C., Cuomo, C.A., Heitman, J., 2014. Analysis of a food-borne fungal pathogen outbreak: virulence and genome of a Mucor circinelloides isolate from yogurt. mBio 5, e01390-14. doi: 10.1128/mBio.01390-14.

Lee, S.C., Li, A., Calo, S., Inoue, M., Tonthat, N.K., Bain, J.M., Louw, J., Shinohara, M.L., Erwig, L.P., Schumacher, M.A., et al., 2015. Calcineurin orchestrates dimorphic transitions, antifungal drug responses and host-pathogen interactions of the pathogenic mucoralean fungus Mucor circinelloides. Mol. Microbiol. 97, 844-865.

Li, C.H., Cervantes, M., Springer, D.J., Boekhout, T., Ruiz-Vázquez, R.M., TorresMartínez, S., Heitman, J., Lee, S.C., 2011. Sporangiospore size dimorphism is linked to virulence of Mucor circinelloides. PLoS Pathog. 7: e1002086. doi: 10.1371/journal.ppat.1002086. 
Lin, Y.L., Ma, L.T., Lee, Y.R., Lin, S.S., Wang, S.Y., Chang, T.T., Shaw, J.F., Li, W.H., Chu, F.H., 2015. microRNA-like small RNAs prediction in the development of Antrodia cinnamomea. PLoS One 10, e0123245. doi: 10.1371/journal.pone.0123245.

MacRae, I.J., Doudna, J.A., 2007. Ribonuclease revisited: structural insights into ribonuclease III family enzymes. Curr. Opin. Struct. Biol. 17, 138-145.

Napoli, C., Lemieux, C., Jorgensen, R., 1990. Introduction of a chimeric chalcone synthase gene into petunia results in reversible co-suppression of homologous genes in trans. Plant Cell 2, 279-289.

Nicolás, F.E., Ruiz-Vázquez, R.M., 2013. Functional diversity of RNAi-associated sRNAs in fungi. Int. J. Mol. Sci. 14, 15348-15360.

Nicolás, F.E., Torres-Martínez, S., Ruiz-Vázquez, R.M., 2003. Two classes of small antisense RNAs in fungal RNA silencing triggered by non-integrative transgenes. EMBO J. 22, 3983-3991.

Nicolás, F.E., de Haro, J.P., Torres-Martínez, S., Ruiz-Vázquez, R.M., 2007. Mutants defective in a Mucor circinelloides dicer-like gene are not compromised in siRNA silencing but display developmental defects. Fungal Genet. Biol. 44, 504-516.

Nicolás, F.E., Moxon, S., de Haro, J.P., Calo, S., Grigoriev, I.V., Torres-Martínez, S., Moulton, V., Ruiz-Vázquez, R.M., Dalmay, T., 2010. Endogenous short RNAs generated by Dicer 2 and RNA-dependent RNA polymerase 1 regulate mRNAs in the basal fungus Mucor circinelloides. Nucleic Acids Res. 38, 5535-5541.

Nicolás, F.E., Vila, A., Moxon, S., Cascales, M.D., Torres-Martínez, S., Ruiz-Vázquez, R.M., Garre, V., 2015. The RNAi machinery controls distinct responses to environmental signals in the basal fungus Mucor circinelloides. BMC Genomics 16, 237. doi: 10.1186/s12864-015-1443-2. 
Park, H., Yu, J., 2012. Genetic control of asexual sporulation in filamentous fungi. Curr. Opin. Microbiol. 15, 669-677.

Pérez, P., Cansado, J., 2010. Cell integrity signaling and response to stress in fission yeast. Curr. Protein Pept. Sci. 11, 680-692.

Romano, N., Macino, G., 1992. Quelling: transient inactivation of gene expression in Neurospora crassa by transformation with homologous sequences. Mol. Microbiol. 6, 3343-3353.

Sarikaya-Bayram, Ö., Palmer, J.M., Keller, N., Braus, G.H., Bayram, Ö., 2015. One Juliet and four Romeos: VeA and its methyltransferases. Front. Microbiol. 6, 1. doi: 10.3389/fmicb.2015.00001.

Sato, S., Suzuki, H., Widyastuti, U., Hotta, Y., Tabata, S., 1994. Identification and characterization of genes induced during sexual differentiation in Schizosaccharomyces pombe. Curr. Genet. 26, 31-37.

Schimek, C., Wöstemeyer, J., 2009. Carotene derivatives in sexual communication of zygomycete fungi. Phytochemistry 70, 1867-1875

Senti, K.A., Brennecke, J., 2010. The piRNA pathway: a fly's perspective on the guardian of the genome. Trends Genet. 26, 409-509

Simmer, F., Buscaino, A., Kos-Braun, I.C., Kagansky, A., Boukaba, A., Urano, T., Kerr, A.R.W., Allshire, R.C., 2010. Hairpin RNA induces secondary small interfering RNA synthesis and silencing in trans in fission yeast. EMBO reports 11, 112-118.

Spellberg, B., Edwards, J.Jr., Ibrahim, A., 2005. Novel perspectives on mucormycosis: pathophysiology, presentation, and management. Clin. Microbiol. Rev. 18, 556-569.

Steyaert, J.M., Weld, R.J., Mendoza-Mendoza, A., Stewart A., 2010. Reproduction without sex: conidiation in the filamentous fungus Trichoderma. Microbiology 156, 2887-2900. 
Trieu, T.A., Calo, S., Nicolás, F.E., Vila, A., Moxon, S., Dalmay, T., Torres-Martínez, S., Garre, V., Ruiz-Vázquez, R.M., 2015. A non-canonical RNA silencing pathway promotes mRNA degradation in basal fungi. PLoS Genet. 11, e1005168. doi: 10.1371/journal.pgen.1005168

Xiao, H., Alexander, W.G., Hammond, T.M., Boone, E.C., Perdue, T.D., Pukkila, P.J., Shiu, P.K.T., 2010. QIP, a protein that converts duplex siRNA into single strands, is required for meiotic silencing by unpaired DNA. Genetics 186, 119-126.

Zhou, J., Fu, Y., Xie, J., Li, B., Jiang, D., Li, G., Cheng, J., 2012. Identification of microRNA-like RNAs in a plant pathogenic fungus Sclerotinia sclerotiorum by highthroughput sequencing. Mol. Genet. Genomics 287, 275-282.

Zong, J., Yao, X., Yin, J., Zhang, D., Ma, H., 2009. Evolution of the RNA-dependent RNA polymerase (RdRP) genes: duplications and possible losses before and after the divergence of major eukaryotic groups. Gene 447, 29-39. 


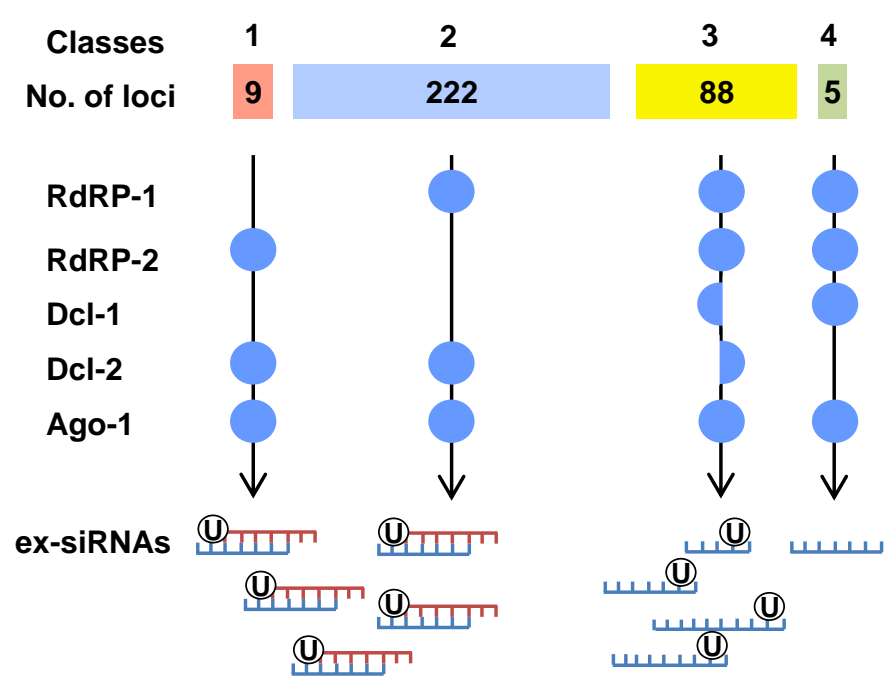

Figure 1 


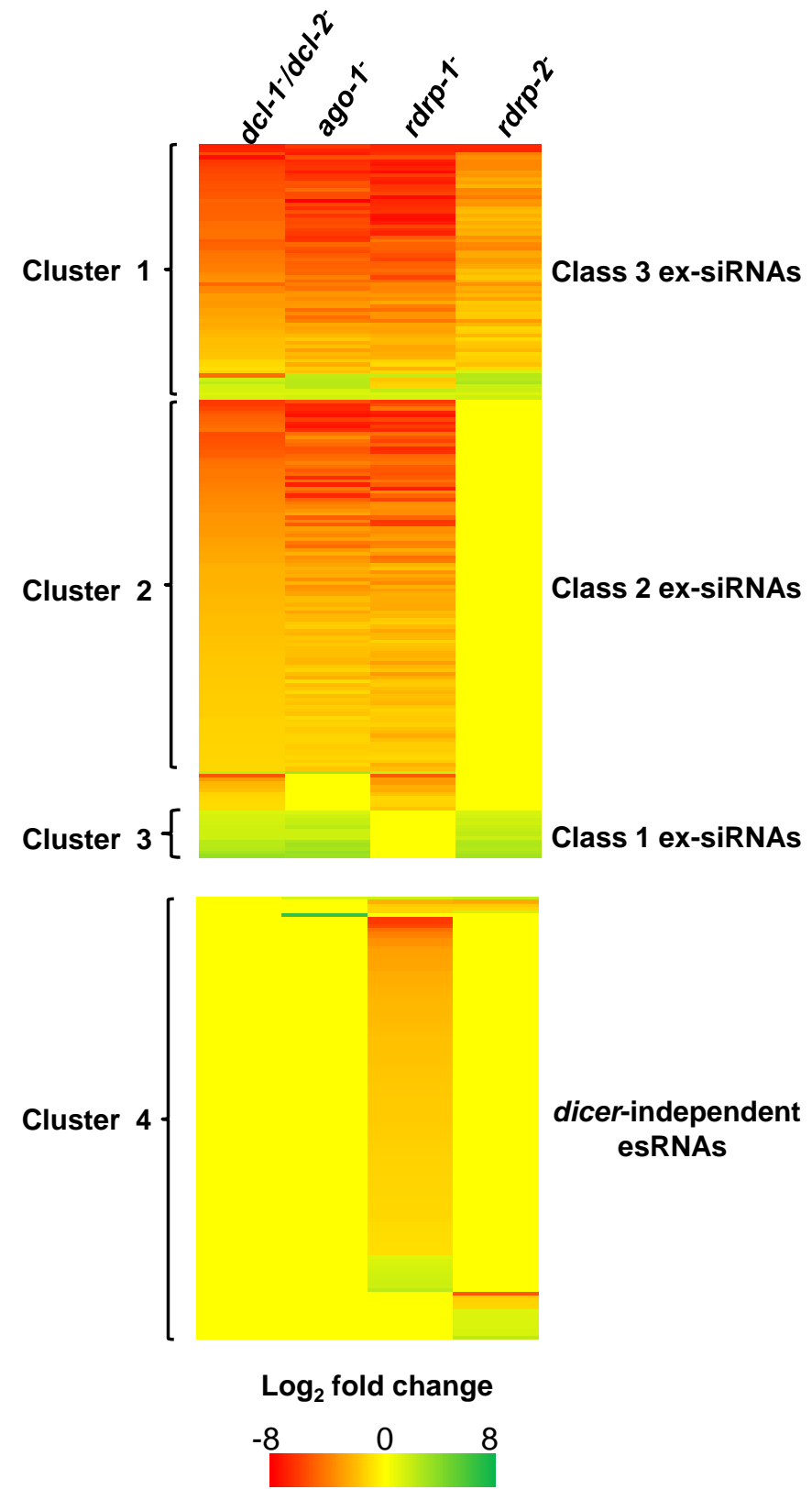

FIGURE 2 


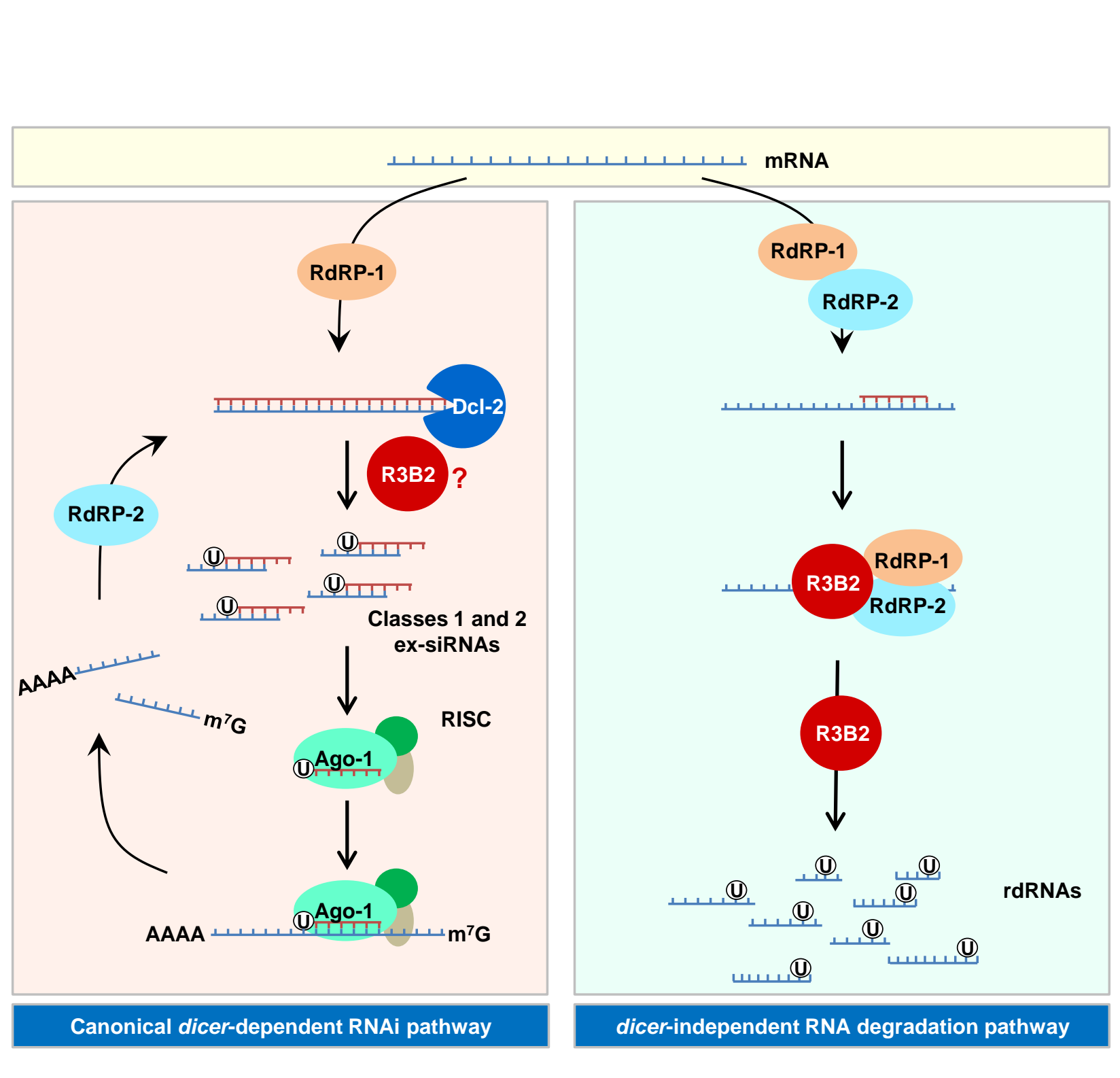

\section{FIGURE 3}

\section{Figure}

\section{FIG}

